\title{
Ultrafast wavelength multiplexed broad bandwidth digital diffuse optical spectroscopy for in vivo extraction of tissue optical properties
}

\author{
Alyssa Torjesen \\ Raeef Istfan \\ Darren Roblyer
}




\title{
Ultrafast wavelength multiplexed broad bandwidth digital diffuse optical spectroscopy for in vivo extraction of tissue optical properties
}

\author{
Alyssa Torjesen, Raeef Istfan, and Darren Roblyer* \\ Boston University, Department of Biomedical Engineering, Boston, Massachusetts, United States
}

\begin{abstract}
Frequency-domain diffuse optical spectroscopy (FD-DOS) utilizes intensity-modulated light to characterize optical scattering and absorption in thick tissue. Previous FD-DOS systems have been limited by large device footprints, complex electronics, high costs, and limited acquisition speeds, all of which complicate access to patients in the clinical setting. We have developed a new digital DOS (dDOS) system, which is relatively compact and inexpensive, allowing for simplified clinical use, while providing unprecedented measurement speeds. The dDOS system utilizes hardware-integrated custom board-level direct digital synthesizers and an analog-to-digital converter to generate frequency sweeps and directly measure signals utilizing undersampling at six wavelengths modulated at discrete frequencies from 50 to $400 \mathrm{MHz}$. Wavelength multiplexing is utilized to achieve broadband frequency sweep measurements acquired at over $97 \mathrm{~Hz}$. When compared to a gold-standard DOS system, the accuracy of optical properties recovered with the dDOS system was within $5.3 \%$ and $5.5 \%$ for absorption and reduced scattering coefficient extractions, respectively. When tested in vivo, the dDOS system was able to detect physiological changes throughout the cardiac cycle. The new FD-dDOS system is fast, inexpensive, and compact without compromising measurement quality. $\odot$ The Authors. Published by SPIE under a Creative Commons Attribution 3.0 Unported License. Distribution or reproduction of this work in whole or in part requires full attribution of the original publication, including its DOI. [DOI: 10.1117/1.JBO.22.3.036009]
\end{abstract}

Keywords: diffuse optics; frequency domain; instrumentation; undersampling.

Paper 160873R received Dec. 22, 2016; accepted for publication Feb. 17, 2017; published online Mar. 10, 2017.

\section{Introduction}

Diffuse optical spectroscopy (DOS) has emerged over the past three decades as an important noninvasive method to characterize optical scattering and absorption and, in turn, calculate chromophore concentrations in thick tissue. ${ }^{1,2}$ Diffuse optical methodologies can be broadly categorized into three distinct measurement types: time domain, frequency domain (FD), and continuous wave $(\mathrm{CW})$. This work focuses on FD-DOS, which allows for the separation of optical scattering and absorption through the measurement of amplitude attenuation and phase delay of photon density waves generated by temporally intensity modulated light sources at the tissue surface. Prior FD-DOS systems have utilized either a single modulation frequency $^{3-9}$ or a sweep of multiple frequencies ${ }^{5-7}$ in the radio frequency (RF) range. Most single modulation frequency systems utilize either homodyne $e^{4,7,8,10}$ or heterodyne ${ }^{3}$ techniques to downconvert the high frequency signal of interest by mixing it with a reference signal. Broad bandwidth DOS instruments, which utilize light sources that are swept through a range of RF frequencies, have typically utilized a benchtop network analyzer to generate and measure signals up to the gigahertz range. ${ }^{1,11}$ FD-DOS devices based around network analyzers have relatively large instrument footprints, and the cost of fabrication can range from approximately $\$ 30,000$ to $\$ 60,000$ (based on our internal cost estimates), limiting access to patients in the clinic. Although some analog systems have been successfully miniaturized, ${ }^{12,13}$ the difficulties involved in RF system design

*Address all correspondence to: Darren Roblyer, E-mail: roblyer@bu.edu potentially limit the adoption of these techniques. Furthermore, FD-DOS instruments often have slow measurement acquisition speeds compared to their $\mathrm{CW}$ counterparts, and although recent improvements have been made in data acquisition rates for single modulation frequency measurements, ${ }^{14}$ data acquisition speeds are limited for broad modulation frequency bandwidth FD-DOS systems. ${ }^{1,10,15}$

We recently demonstrated the feasibility of FD-DOS measurements using all digital signal generation and detection using direct digital synthesizer (DDS) integrated circuits and a 3.6-gigasample/s two-channel analog-to-digital converter (ADC). ${ }^{16}$ These digital components reduced system costs, but measurement acquisition speed was limited due to data transfer bottlenecks between system components. Furthermore, the high sampling rate of the ADC resulted in large datasets and lengthy data transfer times. A second implementation of a digital FDDOS system tested the feasibility of utilizing a slower ADC, sampling at only 25 megasamples/s, and utilizing undersampling techniques. ${ }^{17}$ This substantially reduced system complexity but measurement speed was still a major limitation due to limited data transfer rates. Here, we present a new FD-DOS system that overcomes these past limitations by fully integrating digital signal synthesis and detection to rapidly acquire multiwavelength, multiplexed, broadband frequency sweep measurements at a repetition rate of up to $97 \mathrm{~Hz}$. The digital DOS (dDOS) system was validated relative to a gold-standard network analyzer based DOS system and tested in vivo. This new high-speed, broad-bandwidth, digital, miniaturized DOS system minimizes analog circuitry while reducing cost and device footprint, potentially enabling access to more patients 
in the clinic and expanding the capabilities of this technology, especially for monitoring fast physiological changes.

\section{System Design and Processing}

\subsection{Hardware Integration}

Figure 1 shows the core components and signal/data flow for the new dDOS system. The hardware components of the dDOS system are integrated into a single motherboard that both generates and measures RF signals while communicating and transferring data to the host computer. A development board (ZedBoard, MircoZed Zynq-7010 SoC) was used as the central processing and data storage core for the system. It contains an ARM Cortext-A9 processor and an Artix 7 FPGA, 1 GB of RAM to store data read from the ADC, and a Linux server to transfer data between the board and host computer. The user sets the desired frequency sweep settings in software and the Linux server configures the DDS boards accordingly. The two-channel ADC samples both channels simultaneously and transfers data to the RAM on the MicroZed module via a parallel low voltage differential signal bus. Data are then transferred from the Linux server to the host computer via an Ethernet link at a rate of up to $170 \mathrm{Mbps}$.

\subsection{Light Sources and Modulation}

The dDOS system utilizes NIR laser diodes with wavelengths of $658,690,785,808,830$, and $850 \mathrm{~nm}$ that are housed in thermoelectric cooling modules (Thorlabs, LDM9T). All laser diodes are coupled to $400-\mu \mathrm{m}$ core diameter fibers bundled into a single ferrule. Each laser diode is driven with a DC current, which is mixed with an RF current using a bias-tee to intensitymodulated light. DC current is supplied by an eight-channel, high-stability laser diode controller (ILX Lightwave, LDC390, Newport Corporation). The dDOS system replaces the RF current output functionality of the network analyzer by implementing six DDS chips (AD9910, Analog Devices), each on individual daughterboards. Each DDS can output up to $20 \mathrm{~mA}$ of current modulated up to $400 \mathrm{MHz}$. The RF output of each DDS board is low-pass filtered at $400 \mathrm{MHz}$ and routed to a 7:1 directional coupler. The directional coupler sends the majority of the output power to the bias-tee, and the lesser output from each directional coupler is routed to a power combiner (Mini-Circuits, ZBSC-615+) and measured as a reference signal with the second channel of the ADC.

The dDOS system is capable of utilizing the six individual DDS chips to simultaneously modulate a bank of laser diodes at offset frequencies. The data are then demultiplexed in the FD in postprocessing. A typical dDOS measurement, for example, may sweep through frequencies between 50 and $400 \mathrm{MHz}$ in steps of $1 \mathrm{MHz}$ with the modulation frequency for each wavelength offset by $10 \mathrm{MHz}$. This multiplexing reduces measurement duration to less than $100 \mathrm{~ms} /$ frequency sweep (in the slowest case), allowing for detection of rapid physiological changes. For this work, modulation frequencies between 50 and $300 \mathrm{MHz}$ or between 50 and $400 \mathrm{MHz}$ were used depending on the optical attenuation of the sample and data acquisition rate requirements. The frequency step size can be set between 1 and $7 \mathrm{MHz}$. For wavelengths with modulation sweeps starting above $50 \mathrm{MHz}$, the modulation frequency was incremented up to the maximum frequency then wrapped back to $50 \mathrm{MHz}$ and incremented to the starting frequency. Data were unwrapped such that modulation frequencies appear in increasing order.

\subsection{Detection}

Reflectance mode geometry was used for all dDOS measurements, with the source fiber bundle and active area of an avalanche photodiode (APD) placed directly on the surface of the phantom at a source detector separation of 10 to $30 \mathrm{~mm}$ depending on the specific measurement. An APD module (Hamamatsu C5658) with a $0.5-\mathrm{mm}$ active area, $2.50 \times 105 \mathrm{~V} / \mathrm{W}$ photoelectric sensitivity, and a high band cutoff of $1 \mathrm{GHz}$ was used for most phantom measurements. For in vivo experiments, data were acquired with a 3-mm active area APD.

A 14-bit, two-channel ADC (Texas Instruments, ADS62P49) with a $2 \mathrm{~V}$ peak-to-peak full-scale input voltage was used to

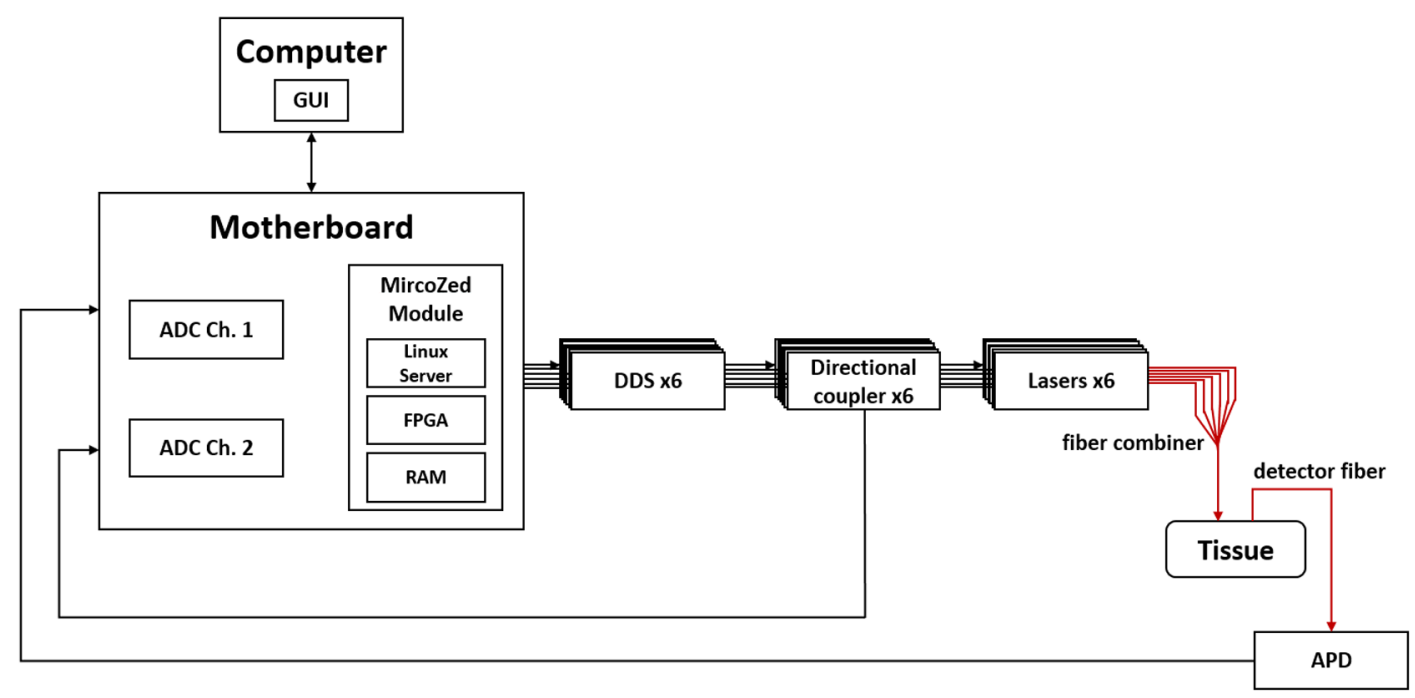

Fig. 1 Schematic overview of dDOS system. GUI, graphical user interface; ADC, analog-to-digital converter; FPGA, field programmable gate array; RAM, rapid access memory; DDS, direct digital synthesizer; APD, avalanche photodiode. 
sample the signal at each channel at 250 MSPS. The electrical output of the APD was high-pass filtered at $41 \mathrm{MHz}$ and routed to the first channel of the ADC, while the combined reference signal from the power combiner was routed to the second channel of the ADC. The user can select the number of samples collected by each channel of the ADC at each frequency step (e.g., $4096,8192,16384 \ldots 2^{n}$ ). Increasing the number of samples per step substantially increases data transfer and processing time. Both channels of the ADC run off of the same clock and are sampled simultaneously.

\subsection{Signal Processing}

All signal processing is performed in MATLAB (R2014b, Mathworks Natick, Massachusetts). Since the two-channel ADC has a sampling rate of $250 \mathrm{MSPS}$, all signals modulated above $125 \mathrm{MHz}$ are aliased. Given that the modulation frequency is known for each wavelength at each frequency step, the signal of interest is easily located in the baseband. The processing algorithm eliminates modulation frequencies that are multiples of the Nyquist frequency and removes any measurements in which multiple wavelengths are aliased to the same frequency in the baseband (e.g., signals at 120 and $130 \mathrm{MHz}$ are both read as $120 \mathrm{MHz}$ and eliminated).

A fast Fourier transform (FFT) with a rectangular window was used at each frequency step to calculate the amplitude and phase for the reference and sample channels. The appropriate frequency bin was located for each wavelength at each modulation frequency step. The magnitude of the FFT of the measurement channel relative to that of the reference channel was considered the raw amplitude value, and, similarly, the phase offset between the measurement and reference channels was considered the raw phase value.

\subsection{Measurement Calibration and Optical Property Extraction}

The raw amplitude and phase represent the amplitude attenuation and phase delay induced both by the tissue and the instrument. In order to remove the instrument response, a measurement was taken on a silicone phantom with known optical properties, where calibration optical properties were obtained using multidistance measurements. An analytical solution to the P1 approximation of the Boltzman transport equation with boundary conditions for semi-infinite geometry was employed as a forward model for the phantom measurement. ${ }^{5}$ Calibration factors for amplitude and phase were determined by ratiometrically or differentially comparing the measured amplitude and phase, respectively, to the theoretical amplitude and phase from the forward model. These calibration factors were then applied to the subsequent raw amplitude and phase measurements in order to obtain calibrated amplitude and phase.

An iterative least-squares fitting algorithm was employed to minimize the error between the forward model calculated and measured calibrated amplitude and phase to extract absorption $\left(\mu_{\mathrm{a}}\right)$ and scattering $\left(\mu_{\mathrm{s}}^{\prime}\right)$ parameters at each wavelength. ${ }^{5}$ Chromophore extinction coefficients were obtained from Zijlstra and Buursma. ${ }^{18}$ The Beer-Lambert law was used to extract chromophore concentrations (oxy- and deoxyhemoglobin) from multiwavelength $\mu_{\mathrm{a}}$ data. ${ }^{13}$

\section{System Characterization}

\subsection{System Overview}

The completed dDOS system is shown in Fig. 2, where the motherboard, DDS daughterboards, MicroZed module, ADC, and RF output connections are pictured. The primary hardware is housed in an enclosure measuring $10 \times 12 \times 6$ in.

\subsection{Signal to Noise}

Signal to noise was measured on a breast-simulating silicone optical phantom. SNR was compared for measurements taken using either a single laser or all six lasers modulated simultaneously. A comparison was also done for measurements taken using either 4096 or 8192 samples. The noise floor was calculated as the average noise level, in $\mathrm{dBc}$, relative to the frequency of interest. Figure 3 shows that when all six lasers were modulated simultaneously, the noise floor was $-46.3 \mathrm{dBc}$, whereas utilizing a single laser yielded a lower noise floor of $-50.1 \mathrm{dBc}$. When increasing the sample length to 8192 samples per frequency step, the noise floor was reduced to -49.2 and $-54.5 \mathrm{dBc}$ for multiplexed and single wavelength measurements, respectively. Although the noise floor was lower with 8192 samples per frequency step, acquisition and data transfer times of these measurements were substantially slower than of those with 4096 samples per frequency step, and there was no improvement in accuracy of optical properties (e.g., the accuracy of 10 measurements on two phantoms with 4096 samples per frequency step was $1.29 \%$ compared to $1.85 \%$ with 8192 samples per frequency step). 4096 samples per frequency step were collected for all subsequent measurements to optimize speed.

\subsection{Accuracy of Optical Property Extractions}

An example of calibrated (black) and fit (red) amplitude and phase measured from 50 to $400 \mathrm{MHz}$ in steps of $1 \mathrm{MHz}$ at each wavelength is shown in Fig. 4. Data were calibrated using a silicone phantom with known optical properties in order to remove the instrument response. Measurements at 658 and $850 \mathrm{~nm}$ have somewhat higher noise levels compared to other wavelengths, likely due to the lower modulation index achieved for these laser diodes.

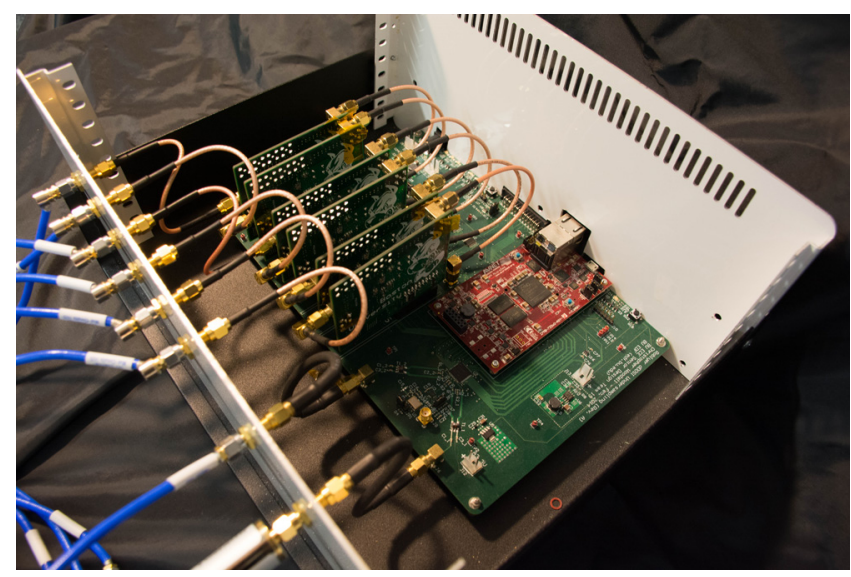

Fig. 2 dDOS core electronics. 


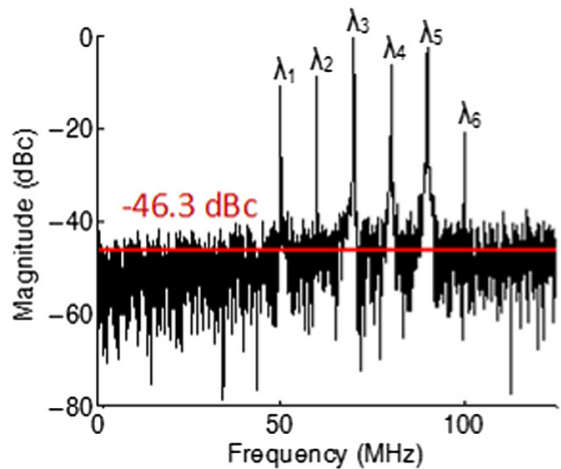

(a)

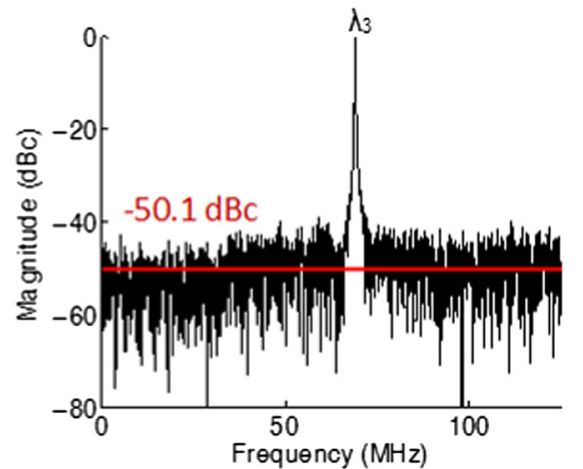

(b)

Fig. 3 Noise floor when collecting 4096 samples per frequency step in (b) a single wavelength measurement or (a) a multiplexed measurement with a $10 \mathrm{MHz}$ offset between wavelengths.
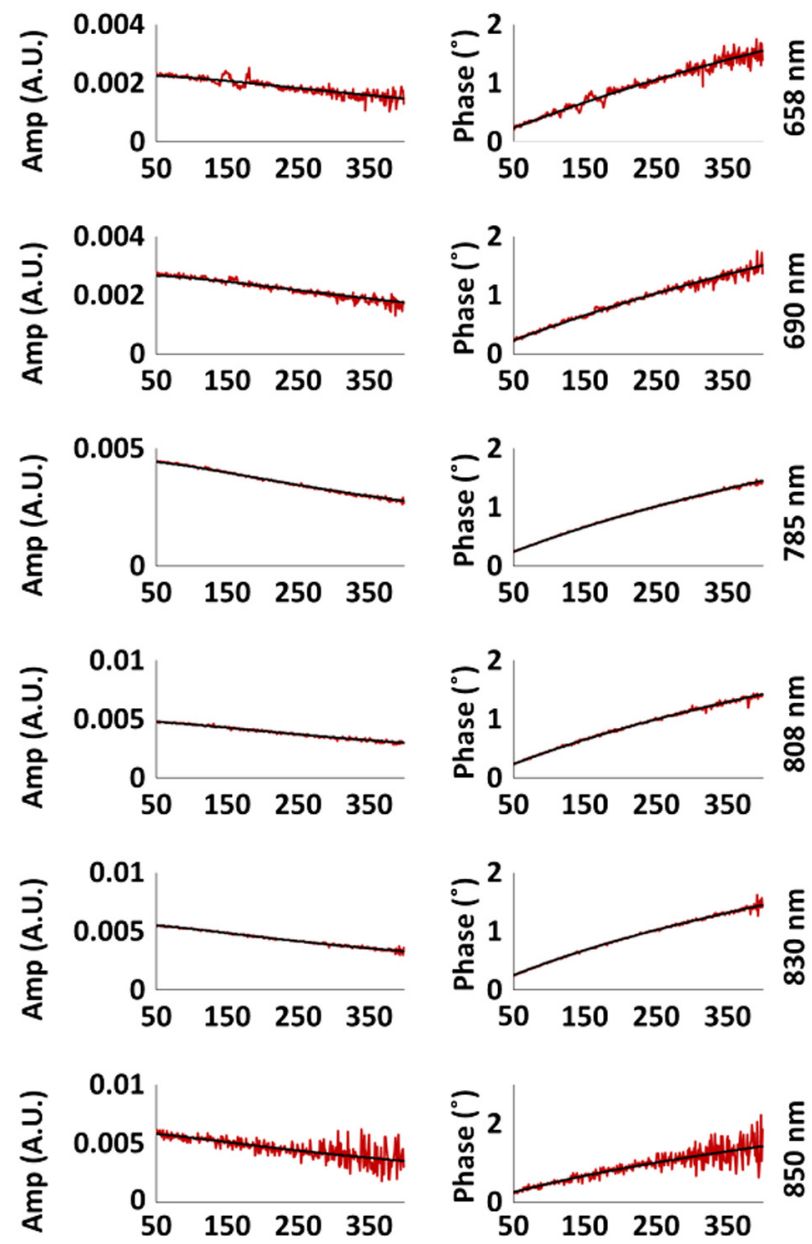

Fig. 4 Amplitude (in arbitrary units, A.U.) and phase measured with dDOS system at each wavelength. Red lines are calibrated data and black lines are model fits.

dDOS optical property extraction accuracy was determined by measuring a set of 10 tissue-simulating silicone phantoms with varying amounts of titanium dioxide and nigrosin, as the scattering and absorbing agents, respectively, using both the dDOS and the network analyzer-based benchtop DOS systems. For each phantom, 10 FD measurements were taken with each system with a $15-\mathrm{mm}$ source-detector separation and all six wavelengths modulated at frequencies between 50 and $400 \mathrm{MHz}$ with $1-\mathrm{MHz}$ frequency steps. Measurements on one highly attenuating phantom at 658, 690, and $850 \mathrm{~nm}$ were excluded due to phase errors resulting from low detected signal levels. The mean of the absolute difference in optical properties for all measured phantoms between the two systems was $5.3 \%$ and $5.5 \%$ for $\mu_{\mathrm{a}}$ and $\mu_{\mathrm{s}}^{\prime}$, respectively; when frequency sweeps from 50 to $300 \mathrm{MHz}$ were used, the mean accuracy in optical properties measured with the two systems was $4.9 \%$ and $6.0 \%$ for $\mu_{\mathrm{a}}$ and $\mu_{\mathrm{s}}^{\prime}$, respectively.

Figure 5 shows scatterplots (top panels) and Bland-Altman plots (bottom panels) for $\mu_{\mathrm{a}}$ and $\mu_{\mathrm{s}}^{\prime}$ measured with the $\mathrm{dDOS}$ and benchtop DOS systems. For the Bland-Altman plots, the differences between optical properties with the dDOS and benchtop system are plotted on the $y$-axis versus average optical properties for each system on the $x$-axis, for each wavelength and phantom. $96 \%$ of $\mu_{\mathrm{a}}$ measurements and $98 \%$ of $\mu_{\mathrm{s}}^{\prime}$ measurements fell within 1.96 standard deviations of the mean difference, indicating acceptable equivalence between the two systems. ${ }^{19} \mu_{\mathrm{a}}$ and $\mu_{\mathrm{s}}^{\prime}$ both tend to skew slightly lower for the dDOS system compared to the benchtop DOS system. $\mu_{\mathrm{a}}$ values had the best agreement between systems when the mean absorption coefficient was $<0.015 \mathrm{~mm}^{-1}$, while $\mu_{\mathrm{s}}^{\prime}$ had the best agreement between systems when the mean reduced scattering coefficient was $>1 \mathrm{~mm}^{-1}$. In healthy breast tissue, $\mu_{\mathrm{a}}$ in the NIR is typically $<0.01 \mathrm{~mm}^{-1}$ and $\mu_{\mathrm{s}}^{\prime}$ in the NIR is typically $>0.8 \mathrm{~mm}^{-1}$. ${ }^{20}$

\subsection{Precision}

The stability of amplitude, phase, and optical properties was characterized during a 2-h drift test. Measurements were taken at $\sim 1$ - min intervals on a silicone optical phantom using $658,690,785,808,830$, and $850 \mathrm{~nm}$ lasers modulated simultaneously in frequency sweeps from 50 to $400 \mathrm{MHz}$ with a $15-\mathrm{mm}$ source-detector separation. Amplitude and phase were examined for the duration of the drift test for the 850-nm laser modulated at $50 \mathrm{MHz}$ and were plotted in Fig. 6. For this wavelength and modulation frequency, the standard deviation of the phase was $0.38 \mathrm{deg}$ and the standard deviation of the amplitude relative to the mean amplitude was $0.81 \%$.

System stability was further analyzed for all wavelengths and modulation frequencies used in the drift test, and results are shown in Table 1. Amplitude and optical property stability were defined as the standard deviation divided by the mean 


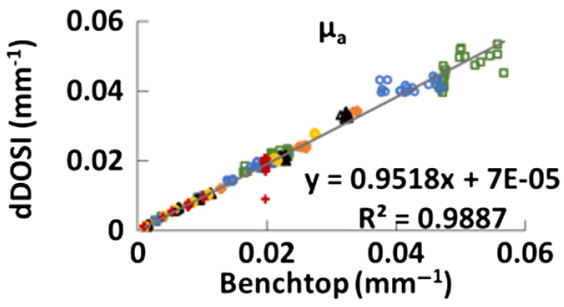

(a)

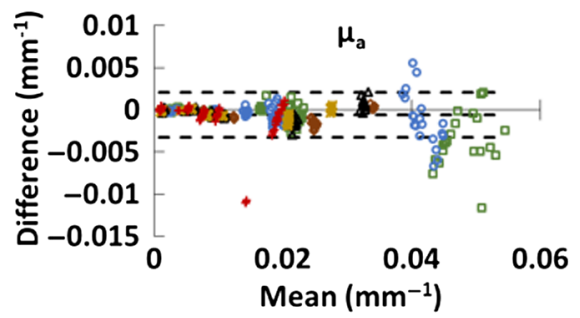

(c)

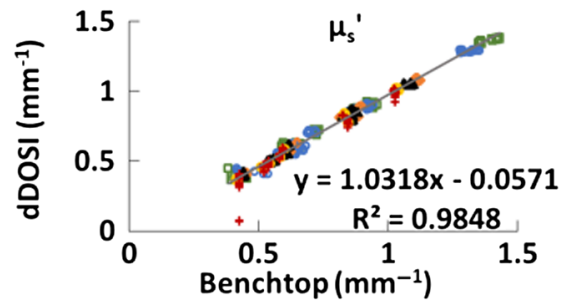

(b)

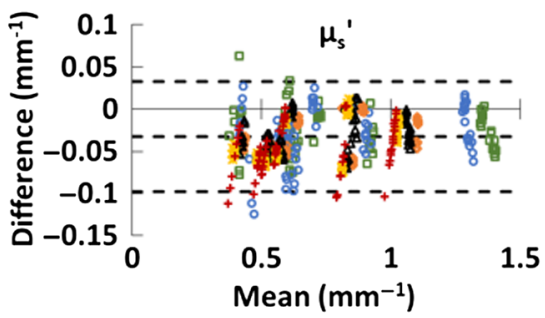

(d)

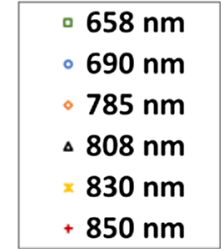

Fig. 5 ( $a$ and b) Scatterplots and (c and d) Bland-Altman plots of optical properties obtained with the dDOS system compared to those obtained with the benchtop system. Top and bottom dashed lines represent 1.96 standard deviations from the mean difference; center dashed lines represent the mean difference.

\section{Amplitude Drift at $50 \mathrm{MHz}$ and $850 \mathrm{~nm}$}

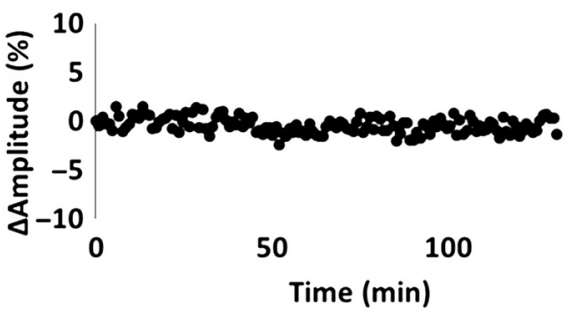

(a)

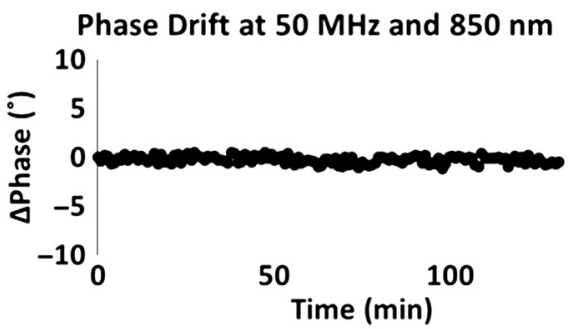

(b)

Fig. 6 2-h drift test showing dDOS system stability. Measurements were taken approximately every minute and show variation from value at the first time point $(t=0)$. (a) Amplitude drift at $50 \mathrm{MHz}$ and $850 \mathrm{~nm}$ and (b) phase drift at $50 \mathrm{MHz}$ and $850 \mathrm{~nm}$.

Table 1 System stability during a 2-h drift test with measurements taken approximately every minute.

Average stability across all wavelengths and modulation frequencies

\begin{tabular}{lc} 
Amplitude & $1.0 \%$ \\
Phase & $1.7 \mathrm{deg}$ \\
$\mu_{\mathrm{a}}$ & $2.0 \%$ \\
$\mu_{\mathrm{s}}^{\prime}$ & $0.8 \%$ \\
\hline
\end{tabular}

at each modulation frequency, averaged for all wavelengths. Phase stability was defined as the standard deviation throughout the drift test averaged for all wavelengths and modulation frequencies. The stability in $\mu_{\mathrm{a}}$ and $\mu_{\mathrm{s}}^{\prime}$ was $2.0 \%$ and $0.8 \%$, respectively.

\subsection{Speed}

Most prior FD-DOS instruments performed frequency sweeps with each wavelength modulated individually in a sequential manner. ${ }^{5,17}$ The dDOS system utilizes wavelength multiplexing, which eliminates switching time between lasers and reduces data acquisition time, resulting in the ability to measure fast physiological changes. The rate at which frequency sweep measurements can be performed is dependent on the range of modulation frequencies, the step size, and the number of samples acquired at each frequency step. When multiplexing six laser wavelengths, sweeping from 50 to $300 \mathrm{MHz}$ with $7 \mathrm{MHz}$ steps, and acquiring 4096 samples per frequency step, the maximum speed achieved for dDOS system was $97.2 \mathrm{~Hz}$. Increasing the frequency sweep range from 50 to $400 \mathrm{MHz}$ reduces the measurement rate to $68.1 \mathrm{~Hz}$. When decreasing the frequency step size to $1 \mathrm{MHz}$, the 50 to $300 \mathrm{MHz}$ sweep can be acquired at $13.9 \mathrm{~Hz}$, and the 50 to $400 \mathrm{MHz}$ sweep can be acquired at $9.8 \mathrm{~Hz}$. These measurement speeds allow for FD-dDOS data acquisition that can capture fast physiological changes, including during the cardiac cycle.

To test the maximum measurement speed attainable with the dDOS system, measurements were also taken with a single modulation frequency. When modulating at only $50 \mathrm{MHz}$, a measurement rate of $2557 \mathrm{~Hz}$ can be achieved. Although this is not the preferred data acquisition mode, because utilizing additional modulation frequencies improves model fit accuracy, it provides a useful comparison to other FD systems, which utilize single modulation frequencies. ${ }^{3,4,8,10,14,21}$

A summary of the performance specifications is given in Table 2. 
Table 2 dDOS performance specifications.

dDOSI performance summary

\begin{tabular}{lc}
\hline SNR (typical) & $46.3 \mathrm{dBc}$ \\
$\mu_{\mathrm{a}}$ accuracy & $5.3 \%$ \\
$\mu_{\mathrm{s}}^{\prime}$ accuracy & $5.5 \%$ \\
Phase stability & $1.7 \mathrm{deg}$ \\
Amplitude stability & $1.0 \%$ \\
Measurement rate (50 to $300 \mathrm{MHz}$ sweeps) & $97.2 \mathrm{~Hz}$ \\
Maximum measurement rate (single frequency) & $2557 \mathrm{~Hz}$ \\
\hline
\end{tabular}

\section{In Vivo Testing}

All in vivo human measurements were carried out under a protocol approved by the Boston University Institutional Review Board.

\subsection{Cuff Occlusion Measurement}

An in vivo cuff occlusion measurement was performed using a blood pressure cuff placed on the upper arm of a male volunteer, while optical measurements were taken on the forearm. The cuff was loosely secured in order to measure baseline molar concentrations of oxyhemoglobin $\left(\mathrm{ctO}_{2} \mathrm{Hb}\right)$ and deoxyhemoglobin (ctHHb). The cuff was then was inflated to $200 \mathrm{mmHg}$ for $\sim 2 \mathrm{~min}$, and then released and data were acquired for $\sim 5$ additional minutes. Frequency sweeps between 50 and $400 \mathrm{MHz}$, with frequency steps of $1 \mathrm{MHz}$, were used to modulate all six laser diodes simultaneously. Measurements were taken approximately every $8 \mathrm{~s}$ for $10 \mathrm{~min}$ and a source-detector separation of $10 \mathrm{~mm}$ was used for each measurement.

Figure 7 shows $\mathrm{ctO}_{2} \mathrm{Hb}$ and $\mathrm{ctHHb}$ throughout the cuff occlusion test. At baseline average, $\mathrm{ctO}_{2} \mathrm{Hb}$ and $\mathrm{ctHHb}$ were 41.6 and $41.2 \mu \mathrm{M}$, respectively. At the time of cuff release, $\mathrm{ctO}_{2} \mathrm{Hb}$ had dropped to $19.3 \mu \mathrm{M}$ and ctHHb had increased to $87.6 \mu \mathrm{M}$. After cuff release, $\mathrm{ctO}_{2} \mathrm{Hb}$ rebounded to a local maximum of $67.7 \mu \mathrm{M}$ and ctHHb dropped to $36.5 \mu \mathrm{M}$, before both chromophore concentrations began to return toward their

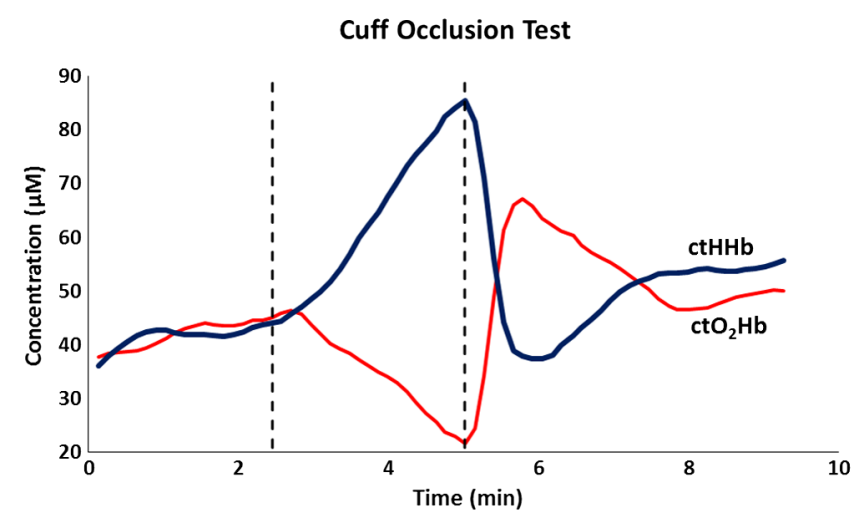

Fig. 7 Forearm cuff occlusion test. Cuff was occluded during time interval between dashed lines. Measurements were taken approximately every $8 \mathrm{~s}$ and a three-point moving average is plotted for each chromophore. baseline values. These results are consistent with reduced arterial supply and vascular drainage during cuff occlusion, followed by a rush of arterial blood into the forearm after cuff release.

\subsection{Rapid In Vivo Measurements}

To test the ability of the dDOS system to measure physiological changes at the cardiac rate, frequency sweep measurements were taken on the finger of a male volunteer. Measurements were taken in reflectance mode on the index finger with a 10 -mm source-detector separation. Frequency sweeps from 50 to $300 \mathrm{MHz}$ with $7 \mathrm{MHz}$ steps were used to modulate all six laser diodes simultaneously. About 350 frequency sweeps were taken with a measurement rate of $97.2 \mathrm{~Hz}$.

An electrocardiogram (ECG) signal was measured simultaneously with the optical signal and synced using an external data acquisition board. Figure 8 shows five-point moving averages of $\mu_{\mathrm{a}}, \mu_{\mathrm{s}}^{\prime}, \mathrm{ctTHb}, \mathrm{ctO}_{2} \mathrm{Hb}$, and ctHHb from the high-speed finger measurement temporally aligned with the ECG. $\mu_{\mathrm{a}}$ values fluctuate temporally with the ECG signal, likely due to increased blood volume in the finger after ventricular contraction. Similarly, ctTHb and ctO ${ }_{2} \mathrm{Hb}$ vary temporally with the ECG signal, while ctHHb shows minimal fluctuation throughout the cardiac cycle. Vertical dashed lines mark the peak of the ECG R-wave, the start of the upstroke of the pulsatile waveform, and the dicrotic notch. There is a 155-ms lag in the upstroke of the $\mu_{\mathrm{a}}$ signal after the R-wave of the ECG, due to the transmission time of the pressure wave to the peripheral vasculature. The delay is often referred to as the pulse transit time and previously shown to range between 200 and 300 ms for photoplethysmographic signals measured in the fingertip. ${ }^{22}$ The dicrotic notch, which is correlated with end of the systolic ejection period, is likely correlated with the dip in $\mu_{\mathrm{a}}$, ctTHb and $\mathrm{ctO}_{2} \mathrm{Hb}$ marked by the rightmost vertical line. In this case, the dichrotic notch appears roughly $303 \mathrm{~ms}$ after the upstroke of the pulsatile waveform, in agreement with other report values in the literature. ${ }^{23,24}$ Fluctuations in $\mu_{\mathrm{a}}$, ctTHb, and $\mathrm{ctO}_{2} \mathrm{Hb}$ appear to occur at the same frequency as the ECG signal, suggesting that the dDOS system is capable of detecting pulsatile, oxygenated, arterial blood flow.

\subsection{Chromophore Maps in Healthy Breast Tissue}

Optical properties of breast tissue were measured on a healthy female volunteer. Measurements were taken with the dDOS system using $658,690,785,808,830$, and $850 \mathrm{~nm}$ lasers with a 28 $\mathrm{mm}$ source-detector separation and modulation frequencies from 50 to $400 \mathrm{MHz}$. 36 point measurements were taken in which the source-detector pair was manually scanned across the right breast in a $6 \times 6 \mathrm{~cm}$ grid pattern with $1 \mathrm{~cm}$ spacing between measurement points. A quadrant of the areolar complex was located at the top left corner of the imaging grid. Optical properties were calculated and chromophore values determined for each data point. Data from the 658- and 850-nm wavelengths were excluded due to noise in the phase measurement at these wavelengths. Figure 9 shows a chromophore map for ctTHb with linear interpolation between measurement points. The chromophore map was superimposed on a 3-D scanned bust model in order to show the approximate relative position of the measurement. There is a region of increased ctTHb at the top left corner of the chromophore map, likely due to increased 


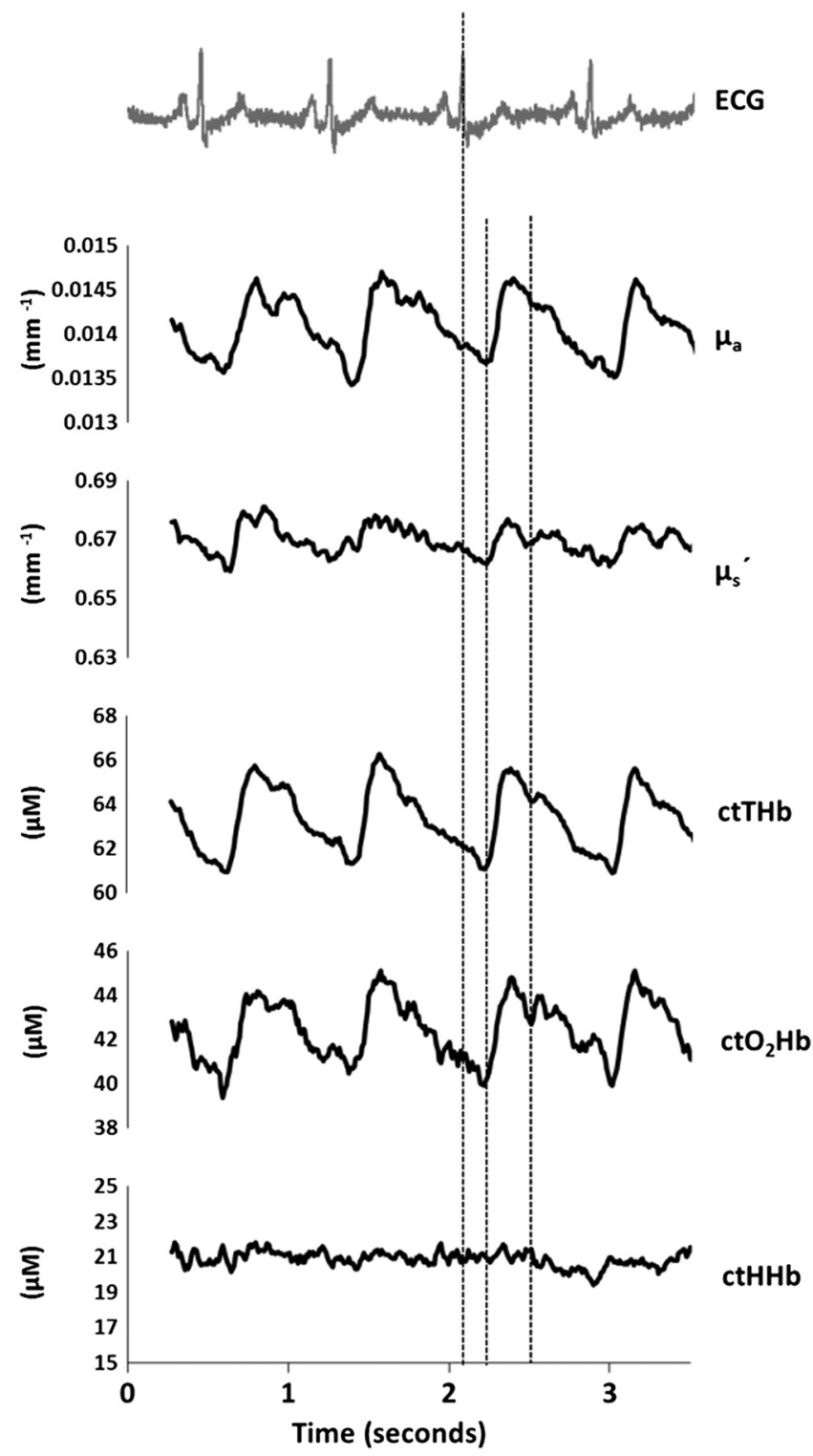

Fig. 8 High-speed dDOS measurements were taken on the index finger. An ECG signal was obtained simultaneously with the optical measurements and temporally aligned with optical property and chromophore values. From top to bottom, plots represent the ECG, $\mu_{\mathrm{a}}, \mu_{\mathrm{s}}^{\prime}, \mathrm{ctTH} \mathrm{b}, \mathrm{ctO}_{2} \mathrm{Hb}$, and $\mathrm{ctHHb}$. The leftmost vertical dashed line represents the approximate time of the peak of the ECG Rwave, the middle dashed line represents the approximate time of the start of the upstroke of the optical signal following the arterial transit time, and the rightmost dashed line represents the approximate timing of the dicrotic notch based on visual inspection.

blood supply to the areola as compared to standard breast tissue, which has been observed in prior DOS studies. ${ }^{25}$

\section{Discussion}

A new dDOS system was developed to increase access to patients, while adding new capabilities in measurement speed. Traditional network analyzer-based FD-DOS systems are large and costly, and miniaturization of analog RF circuits is technically challenging. The hardware-integrated dDOS system developed here utilizes direct digital signal generation and detection and wavelength multiplexing, with frequency sweep measurements rates up to $97 \mathrm{~Hz}$, allowing rapid physiological changes
Total hemoglobin concentration

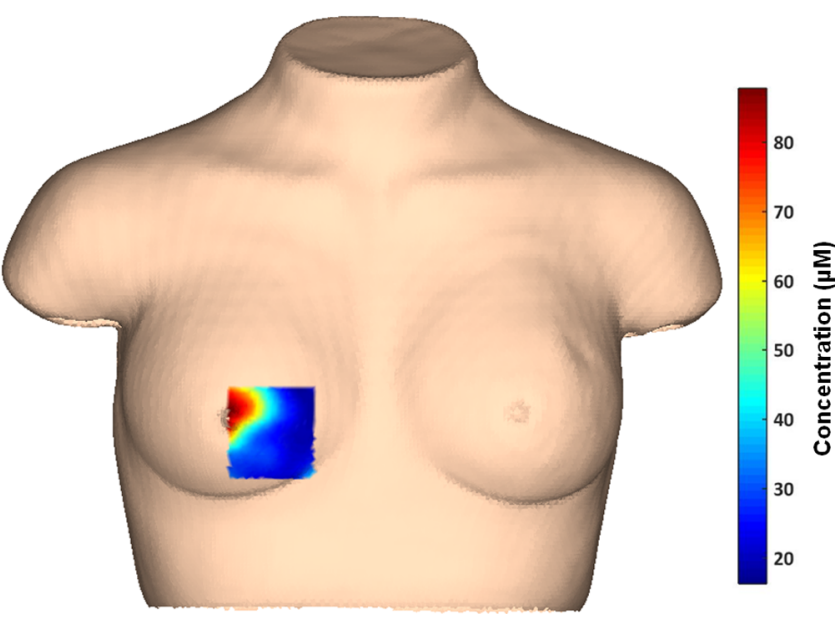

Fig. $9 \mathrm{ctTHb}$ measured on the right breast of a healthy volunteer. A quadrant of the nipple is located in the lower left corner of the image.

to be detected well above the cardiac rate. Previous systems achieving high sampling rates have utilized a small number of discrete modulation frequencies and wavelengths, ${ }^{14,21}$ whereas the dDOS system sweeps a range of hundreds of modulation frequencies and utilizes six discrete wavelengths.

The dDOS system performance is comparable to previous systems. For example, Pham et al. reported an accuracy of $\pm 5 \%$ and $\pm 3 \%$ for $\mu_{\mathrm{a}}$ and $\mu_{\mathrm{s}}^{\prime}$, respectively, whereas the $\mathrm{dDOS}$ system had an accuracy of $\pm 5.3 \%$ and $\pm 5.5 \%$ for the same parameters. The dDOS design has similarities to that of the modular digital FD diffuse optical monitor developed by Durduran et al., which utilized digital signal synthesis and a dual-channel ACD to sample and downconvert the signal from three laser wavelengths at a single modulation frequency, ${ }^{21}$ however, our design differs in that we use six multiplexed laser wavelengths modulated at hundreds of discrete frequencies in the RF range. Zimmerman et al. recently developed a FD NIR high speed imaging system that utilized two wavelengths, two modulation frequencies, and an array of detectors; with this system, a measurement rate of $90 \mathrm{~Hz}$ was achieved, compared to the $97-\mathrm{Hz}$ measurement rate achieved in the dDOS system with six wavelengths and a sweep of modulation frequencies. ${ }^{14}$

In the future, the portability of the system can be further improved by implementing miniaturized fiber-coupled laser diodes and miniaturized current driver modules that can be housed in a portable device enclosure. This will help to more easily measure patients in a clinical setting for a range of applications, such as chemotherapy monitoring or rapid hemodynamic analysis. The speed of the system also opens the possibility of monitoring rapid physiological changes at the cardiac rate, which may be useful in monitoring hemodynamics related to conditions, such as peripheral artery disease.

\section{Conclusions}

We have developed an inexpensive clinical FD-DOS platform based on DDSs and ADCs implemented on custom hardware-integrated PCBs. The system has high stability and accuracy in phantom measurements and is capable of detecting rapid physiological fluctuations above the cardiac rate using six wavelengths and a sweep of modulation frequencies. The dDOS 
system may improve access to patients while helping to open new areas of investigation.

\section{Disclosures}

The authors have no conflicts of interest.

\section{Acknowledgments}

We would like to thank Dr. Eric Hazen and the Boston University Electronics Design Facility for their ongoing support with hardware and firmware development. The authors gratefully acknowledge funding from the American Cancer Society (Grant No. RSG-14-014-01-CCE) and the Department of Defense (Award No. W81XWH-15-1-0070).

\section{References}

1. B. J. Tromberg et al., "Non-invasive measurements of breast tissue optical properties using frequency-domain photon migration," Philos. Trans. R. Soc. Lond. B. Biol. Sci. 352, 661-668 (1997).

2. B. J. Tromberg et al., "Optical property measurements in turbid media using frequency domain photon migration," Proc. SPIE 1525, 52-58 (1991).

3. T. O. McBride et al., "A parallel-detection frequency-domain near-infrared tomography system for hemoglobin imaging of the breast in vivo," Rev. Sci. Instrum. 72, 1817-1824 (2001).

4. J. P. Culver et al., "Three-dimensional diffuse optical tomography in the parallel plane transmission geometry: evaluation of a hybrid frequency domain/continuous wave clinical system for breast imaging," Med. Phys. 30, 235-247 (2003).

5. T. H. Pham et al., "Broad bandwidth frequency domain instrument for quantitative tissue optical spectroscopy," Rev. Sci. Instrum. 71, 25002513 (2000)

6. U. J. Netz, J. Beuthan, and A. H. Hielscher, "Multipixel system for gigahertz frequency-domain optical imaging of finger joints," Rev. Sci. Instrum. 79, 034301 (2008).

7. Y. S. Yang et al., "Low-cost frequency-domain photon migration instrument for tissue spectroscopy, oximetry, and imaging," Opt. Eng. 36 1021-1569 (1997).

8. G. Yu et al., "Frequency-domain multiplexing system for in vivo diffuse light measurements of rapid cerebral hemodynamics," Appl. Opt. 42, 2931-2939 (2003).

9. Y. Zhao et al., "Portable, parallel 9-wavelength near-infrared spectral tomography (NIRST) system for efficient characterization of breast cancer within the clinical oncology infusion suite," Biomed. Opt. Express 7, 2186-2201 (2016).
10. B. Pogue et al., "Instrumentation and design of a frequency-domain diffuse optical tomography imager for breast cancer detection," Opt. Express 1, 391-403 (1997).

11. S. J. Madsen et al., "Keywords: frequency-domain photon migration, optical properties, diode laser, network analyzer," Proc. SPIE 2389, 257-263 (1995)

12. K.-S. No et al., "Design and testing of a miniature broadband frequency domain photon migration instrument," J. Biomed. Opt. 13, 050509 (2009).

13. T. D. O'Sullivan et al., "Diffuse optical imaging using spatially and temporally modulated light," J. Biomed. Opt. 17, 071311 (2012).

14. B. B. Zimmermann et al., "Frequency domain near-infrared multiwavelength imager design using high-speed, direct analog-to-digital conversion," J. Biomed. Opt. 21, 016010 (2016).

15. J. Jung, R. Istfan, and D. Roblyer, "Note: a simple broad bandwidth undersampling frequency-domain digital diffuse optical spectroscopy system," Rev. Sci. Instrum. 85, 076108 (2014).

16. D. Roblyer et al., "Feasibility of direct digital sampling for diffuse optical frequency domain spectroscopy in tissue," Meas. Sci. Technol. 24, 045501 (2013).

17. J. Jung, R. Istfan, and D. Roblyer, "Note: a simple broad bandwidth undersampling frequency-domain digital diffuse optical spectroscopy system," Rev. Sci. Instrum. 85, 076108 (2014).

18. W. G. Zijlstra and A. Buursma, "Spectrophotometry of hemoglobin: absorption spectra of bovine oxyhemoglobin, deoxyhemoglobin, carboxyhemoglobin, and methemoglobin," Comput. Biochem. Physiol. 118, 743-749 (1997).

19. J. M. Bland and D. G. Altman, "Statistical methods for assessing agreement between two methods of clinical measurement," Int. J. Nurs. Stud. 47, 931-936 (2010).

20. N. Shah et al., "Spatial variations in optical and physiological properties," J. Biomed. Opt. 9, 534-540 (2004).

21. U. M. Weigel et al., "A new, modular frequency domain diffuse optical monitor in the digital domain," in Digital Holography and ThreeDimensional Imaging, Optical Society of America (2012).

22. J. Yong, A. Foo, and C. S. Lim, "Pulse transit time based on piezoelectric," J. Clin. Monit. Comput. 20, 185-192 (2006).

23. W. Lee et al., "Systolic time intervals derived from electrocardiographic gated intra- renal artery Doppler waveform associated with left ventricular systolic function," Sci. Rep. 6, 29293 (2016).

24. M. Vyas et al., "Augmentation index and central aortic stiffness in middle-aged to elderly individuals," Am. J. Hypertens. 20, 642-647 (2007).

25. D. Roblyer et al., "Optical imaging of breast cancer oxyhemoglobin flare correlates with neoadjuvant chemotherapy response one day after starting treatment," Proc. Natl. Acad. Sci. U. S. A. 108, 1462614631 (2011)

Biographies for the authors are not available. 